\title{
CHARACTERIZATIONS OF GRAPHS HAVING LARGE PROPER CONNECTION NUMBERS
}

\author{
Chira Lumduanhom ${ }^{1}$ \\ Department of Mathematics \\ Srinakharinwirot University \\ Sukhumvit Soi 23, Bangkok, 10110, Thailand \\ Elliot Laforge AND Ping Zhang \\ Department of Mathematics \\ Western Michigan University \\ Kalamazoo, MI 49008, USA \\ e-mail: ping.zhang@wmich.edu
}

\begin{abstract}
Let $G$ be an edge-colored connected graph. A path $P$ is a proper path in $G$ if no two adjacent edges of $P$ are colored the same. If $P$ is a proper $u-v$ path of length $d(u, v)$, then $P$ is a proper $u-v$ geodesic. An edge coloring $c$ is a proper-path coloring of a connected graph $G$ if every pair $u, v$ of distinct vertices of $G$ are connected by a proper $u-v$ path in $G$, and $c$ is a strong proper-path coloring if every two vertices $u$ and $v$ are connected by a proper $u-v$ geodesic in $G$. The minimum number of colors required for a proper-path coloring or strong proper-path coloring of $G$ is called the proper connection number $\operatorname{pc}(G)$ or strong proper connection number $\operatorname{spc}(G)$ of $G$, respectively. If $G$ is a nontrivial connected graph of size $m$, then $\operatorname{pc}(G) \leq \operatorname{spc}(G) \leq m$ and $\operatorname{pc}(G)=m$ or $\operatorname{spc}(G)=m$ if and only if $G$ is the star of size $m$. In this paper, we determine all connected graphs $G$ of size $m$ for which $\operatorname{pc}(G)$ or $\operatorname{spc}(G)$ is $m-1, m-2$ or $m-3$.
\end{abstract}

Keywords: edge coloring, proper-path coloring, strong proper-path coloring.

2010 Mathematics Subject Classification: 05C15, 05C38, 05C75.

\section{REFERENCES}

\footnotetext{
${ }^{1}$ Research supported by a New Researcher Grants sponsored by Ministry of Science \& Technology, Thailand.
} 
[1] E. Andrews, E. Laforge, C. Lumduanhom and P. Zhang, On proper-path colorings in graphs, J. Combin. Math. Combin. Comput., to appear.

[2] G. Chartrand, G.L. Johns, K.A. McKeon and P. Zhang, Rainbow connection in graphs, Math. Bohem. 133 (2008) 85-98.

[3] G. Chartrand, L. Lesniak and P. Zhang, Graphs \& Digraphs, 5th Edition (Chapman \& Hall/CRC, Boca Raton, FL, 2010).

[4] G. Chartrand and P. Zhang, Chromatic Graph Theory (Chapman \& Hall/CRC, Boca Raton, FL, 2008). doi:10.1201/9781584888017

[5] A.B. Ericksen, A matter of security, Graduating Engineer \& Computer Careers (2007) 24-28.

[6] X.L. Li and Y.F. Sun, Rainbow Connections of Graphs (Springer, Boston, MA, 2012). doi:10.1007/978-1-4614-3119-0

[7] X.L. Li, Y.F. Sun and Y. Zhao, Characterization of graphs with rainbow connection number $m-2$ and $m-3$, Australas. J. Combin. 60 (2014) 306-313.

Received 15 October 2014

Revised 5 August 2015

Accepted 10 August 2015 